\title{
La presse française face à la guerre civile grecque
} (1946-1949)

The French press and the Greek civil War (1946-1949)

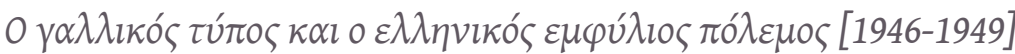

\section{Athanasia Balta}

\section{OpenEdition}

Journals

Édition électronique

URL : https://journals.openedition.org/ceb/3986

DOI : $10.4000 / c e b .3986$

ISSN : 2261-4184

Éditeur

INALCO

Édition imprimée

Pagination : 199-208

ISBN : 978-2-85831-205-4

ISSN : 0290-7402

Référence électronique

Athanasia Balta, «La presse française face à la guerre civile grecque (1946-1949) », Cahiers

balkaniques [En ligne], 41 | 2013, mis en ligne le 19 mai 2013, consulté le 06 juillet 2021. URL : http:// journals.openedition.org/ceb/3986; DOI : https://doi.org/10.4000/ceb.3986

Ce document a été généré automatiquement le 6 juillet 2021

\section{(c) (†) \&}

Cahiers balkaniques est mis à disposition selon les termes de la Licence Creative Commons Attribution - Pas d'Utilisation Commerciale 4.0 International. 


\title{
La presse française face à la guerre civile grecque (1946-1949)
}

\author{
The French press and the Greek civil War (1946-1949)

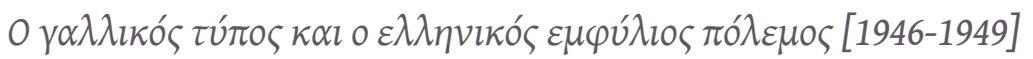

\section{Athanasia Balta}

1 Très peu de temps après la fin de la Seconde Guerre mondiale, la guerre civile grecque est l'un des premiers épisodes de la guerre froide qui se dessine et qui, en Grèce, devient extrêmement brûlante. Les événements de Grèce occupent l'attention de la presse française dès le début de l'année 1946, alors que le pays glisse progressivement vers la guerre civile.

2 Cette communication porte sur la façon dont cette guerre a été envisagée par tous les grands quotidiens parisiens et par un grand nombre de revues et de périodiques couvrant tout le phasme politique. Comme il est impossible de suivre l'évolution de l'information et des positions de diverses tendances de la presse dans toutes ses nuances, on a tenté de dresser ici un bilan et de résumer quelques conclusions générales ${ }^{1}$.

3 Quel intérêt pouvait porter la presse française à des faits ayant lieu à l'autre bout de l'Europe, dans un temps où la France elle-même cherchait à se rétablir et à s'adapter aux problèmes de l'après-guerre? Comment la presse française a-t-elle couvert les événements en Grèce et comment a-t-elle inséré cet événement dans le contexte international ? Répondre à cette question signifie essayer de comprendre, de ce point de vue précis, comment les Français vivent cette première période de rupture et de confrontation entre les deux blocs.

\section{De l'« affaire grecque » à la guerre civile grecque (début 1946-mars 1947)}

4 L'année 1946 voit la Grèce à la «Une » de l'ensemble de la presse à l'occasion de la discussion de la question grecque au Conseil de Sécurité de l'ONU. Du printemps à 
l'automne 1946, tous les journaux donnent l'impression de croire à un règlement démocratique du problème, aussi ont-ils attaché un intérêt particulier aux deux scrutins, celui des élections, en mars, et celui du plébiscite sur le retour du roi Georges, en septembre. La presse de gauche, même après les deux scrutins qu'elle considère comme "truqués ", continue à espérer en un gouvernement d'union nationale auquel la gauche devrait absolument participer. Pour la presse de droite, la solution a été donnée par les résultats des élections et du plébiscite, mais la tension dans le pays continue et s'accentue à cause de la politique insurrectionnelle des communistes grecs. La présence britannique est jugée comme indispensable pour le maintien de l'ordre et le retour à la stabilité. Le Monde en reste à une vision du problème purement politique : la solution de la crise ne résultera que d'« une politique modérée et prudente $»^{2}$, de la part du nouveau gouvernement de K. Tsaldaris, du roi Georges et des libéraux du centre de Th. Sophoulis, à qui le journal attache une grande importance. La presse communiste, au contraire, ignore les partis et les chefs politiques bourgeois et quand elle les mentionne, elle le fait pour les dénoncer en tant que collaborateurs du Premier ministre Tsaldaris, traité de «monarchofasciste».

5 Durant les mois de septembre et d'octobre, les nouvelles de Grèce sont de plus en plus inquiétantes. La «crise » devient « prodrome de guerre civile » et peu après « guerre civile ». En France, où depuis quelques mois la gauche n'est plus majoritaire et où le tripartisme est disloqué, et dans un climat de tensions croissantes dans les relations internationales, la plupart des journaux cherchent à comprendre, tracent les causes de cette lutte fratricide qui ensanglante la Grèce dans le lourd héritage du passé récent de l'Occupation et de la Résistance, dans le malaise économique, mais aussi dans les complications internationales.

6 Les premiers envoyés spéciaux des journaux Le Monde, Combat, Ce Soir, Le Figaro et des revues Carrefour, L'Action et Gavroche, qui arrivent en Grèce dès le début de l'année, ne se limitent pas à informer leurs lecteurs sur l'aspect politique de la crise, ils essaient de leur faire ressentir l'ambiance quotidienne dans laquelle vit le peuple à Athènes et dans les provinces, où règnent la peur et la misère. À partir d'octobre les andartès passent à l'attaque et la Macédoine devient le théâtre des combats. Dans la confusion générale provoquée par des informations et une propagande contradictoires, les positions de journaux deviennent, elles, de plus en plus claires. La question cruciale de l'hiver, au début de 1947, est l'identité des partisans : qui sont les rebelles? Patriotes pour la presse communiste, terroristes et criminels pour les journaux conservateurs comme L'Aurore ou L'Époque, les « andartès » occupent de jour en jour plus d'espace dans tous les journaux.

7 Pendant cette première période, l'aspect international du problème grec consiste en ce qu'il représente un danger pour la paix dans les Balkans et donc, pour la paix mondiale. La discussion de la question grecque au Conseil de Sécurité de l'ONU, en janvier et en août 1946, donne à l'ensemble de la presse l'occasion d'exprimer son inquiétude face à l'éventualité d'une nouvelle guerre, à l'exception de l'Humanité qui cherche plutôt à soutenir les thèses de la délégation soviétique.

8 À la veille de la doctrine Truman, la presse apparaît déjà divisée quant à l'approche de la question grecque. Pour les journaux de droite tels L'Aurore, L'Époque ainsi que L'Aube, il n'y a pas de causes internes à la crise grecque. Le chaos qui ne cesse de s'aggraver est dû aux menées soviétiques par l'intermédiaire de ses agents, les communistes grecs. Aux antipodes d'une telle opinion, la presse communiste réduit tout à l'impérialisme 
britannique. Des points de vue plus nuancés sont exprimés par deux journaux de la gauche non communiste, Le Populaire de Paris et Combat, par Le Figaro, qui adopte une position plutôt modérée, ainsi que par les envoyés spéciaux du journal Le Monde, dont les points de vue ne s'accordent pas toujours avec ceux exprimés par le journal dans les bulletins de l'étranger.

\section{De la doctrine Truman à « l'affaire Tito ${ }^{3}$ (mars 1947- juin 1948)}

9 Pendant cette période la division de l'Europe devient définitive. La réponse communiste à la doctrine Truman et au Plan Marshall, donnée par la création du Kominform (Bureau d'information des Partis communistes) en septembre 1947, confirme l'existence de deux camps opposés. Une série d'événements importants, le coup de Prague, en février 1948, le blocus de Berlin et l'exclusion des hérétiques yougoslaves de Tito du Kominform, dans l'été 1948, accentuent la détérioration du climat international. L'Ouest se laisse de plus en plus glisser dans l'angoisse et la peur du communisme.

10 Au printemps 1947 il est clair que l'importance de ce conflit fratricide dépasse les frontières grecques. L'information, désormais plus riche, et les articles dans les journaux jusqu'alors plutôt indifférents témoignent d'un intérêt de plus en plus grandissant. L'Aube, Le Figaro et Carrefour envoient en Grèce leurs envoyés spéciaux, et L'Humanité, son premier correspondant, Germain Rigal, à qui Markos accorde sa première interview, parue à la Une comme « une exclusivité mondiale ${ }^{4}$. Chaque jour la Grèce est à la "Une" de tous les journaux, surtout à l'occasion des travaux de la Commission spéciale de l'ONU, au printemps et pendant l'été, au moment des opérations militaires en Épire et de l'intensification des mesures de répression contre la gauche grecque. La presse communiste, L'Humanité en tête, dénonce la doctrine Truman et l'intervention américaine en Grèce. La presse de la gauche communiste et non communiste proteste vivement contre la répression, les arrestations massives et la violation des libertés démocratiques par le gouvernement grec. Dans Le Populaire de Paris, Léon Blum lui-même exprime la solidarité socialiste. Dans les publications de l'organe de la SFIO se fait jour un difficile équilibre : le journal désapprouve l'action des andartès de l'A.D. ${ }^{5}$, «simples pions dans le jeu de l'impérialisme bolchevique » et dénonce en même temps «le régime fasciste » d'Athènes ${ }^{6}$. La presse de droite de toute tendance s'exprime en faveur de l'intervention américaine, considérée comme le seul moyen de protéger la Grèce et toute la civilisation occidentale contre l'expansionnisme et la barbarie soviétique. Entre l'attitude modérée du Figaro et l'anticommunisme dur de L'Aurore par exemple, il y a un décalage dans le style, le vocabulaire, la disposition d'esprit et la qualité d'information, mais il y a également un dénominateur commun : la conviction que la guerre civile est provoquée par l'action traître des communistes grecs, soutenus moralement et matériellement par l'URSS.

11 Entre-temps, la France, confrontée à une agitation sociale croissante, fait son entrée dans la guerre froide. Depuis mai 1947, il n'y a plus de ministres communistes dans le gouvernement Ramadier et le PCF se trouve désormais dans l'opposition. Ce grand tournant de sa politique après le départ du gouvernement, la formation du Kominform et les grandes grèves d'automne 1947 aggravent la division au sein de la société française. L'Humanité fait de plus en plus usage de l'exemple grec pour lancer des 
attaques rigoureuses contre le gouvernement français en matière de politique étrangère.

12 À cette époque, de septembre 1947 à juin 1948, la guerre civile grecque entre dans sa phase la plus décisive. En décembre 1947 la constitution du GDP provoque une grande sensation et des réactions diverses. L'Humanité publie la nouvelle de la formation « du gouvernement des montagnes » en exprimant sa certitude qu'il sera reconnu par les pays communistes et même par l'ONU. Cette reconnaissance ne viendra jamais ni de l'URSS ni des démocraties populaires ${ }^{7}$. Une véritable «vague » de journalistes, envoyés par tous les grands journaux, vient confirmer l'importance particulière attribuée aux événements grecs à la lumière des nouvelles données de la situation internationale.

L'intérêt du Monde pour les événements en Grèce se traduit par le nombre extrêmement abondant des publications. En automne 1947, le grand quotidien est persuadé d'une part de l'action traîtresse des « rebelles communistes » et, d'autre part, de l'intention sincère des États-Unis d'aider la Grèce en danger. Après la formation du GDP, le journal exprime la conviction qu'il s'agit d'« une nouvelle manifestation de l'activité du Kominform $»^{8}$. Le journaliste du Monde, Marc Marceau, arrive à Athènes en janvier 1948, et il est le seul correspondant permanent français pendant toute la période. Ses correspondances, sous le titre "Les lettres d'Athènes» présentent un intérêt particulier. Marceau est d'accord avec la ligne officielle de son journal sur le fait que cette guerre est provoquée par l'expansionnisme soviétique, par l'intermédiaire des pays satellites balkaniques. Mais ses jugements sur les facteurs intérieurs du problème sont extrêmement différents : tout en restant anticommuniste et hostile à la gauche grecque, il accuse les gouvernements grecs d'avoir suivi une politique incohérente et irresponsable et il rejette tous les politiciens de la droite et du centre comme incapables.

14 D'un grand intérêt sont aussi les reportages de l'envoyée spéciale de l'Humanité, Simone Téry. Elle arrive en "Grèce libre ", dans la région du nord de la Grèce, en octobre 1947, comme "envoyée spéciale de L'Humanité à l'armée de Markos». Ses reportages sont publiés sous le titre général «Les hommes de cœur sont plus forts que les dollars », du 19 décembre 1947 au 14 janvier 1948. Très impressionnée par le moral des combattants et combattantes de l'Armée démocratique et par la personnalité de son chef, le général Markos Vafiadis, très remuée aussi par le climat d'affection et de fraternité manifesté à son égard, elle ne cache pas son émotion et, avec beaucoup de lyrisme, elle donne l'image idyllique d'une armée de héros. Parallèlement elle ne manque pas de «tirer les leçons » politiques nécessaires :

...Quelle leçon que la Grèce pour nous, Français, et pour tous les peuples que veut coloniser l'impérialisme américain, quelle leçon et quelle exemple !...En Grèce, j’ai vu ce que, si nous le laissons faire, le parti américain prépare pour la France; le retour des années du malheur, les traîtres revenus au gouvernement ${ }^{9} .$.

C'est pendant l'été 1947 que la guerre d'Espagne est évoquée comme parallèle historique. La comparaison qui montre les points communs entre les deux cas est formulée pour la première fois dans l'Humanité ${ }^{10}$ et réapparaît à maintes reprises comme un thème stable dans la presse communiste. La presse de droite fait aussi usage de la guerre civile espagnole comme référence et expérience historique qui devrait convaincre gouvernements et opinions publiques du rôle joue par l'Union Soviétique en Grèce ${ }^{11}$. 
16 Entre les deux versions d'interprétation et de propagande, communiste et anticommuniste, il existe une tendance mineure exprimée par une petite partie de la presse et certains intellectuels non communistes. Les journaux Combat, Franc-Tireur, la revue Esprit et jusqu'à un certain point Le Populaire de Paris, se tiennent à égale distance entre les deux grandes puissances en ce qui concerne l'aspect extérieur des événements de Grèce et, en même temps, jugent sévèrement le gouvernement grec pour sa politique de répression contre la gauche. Cette attitude peut être considérée comme l'une des premières manifestations du mouvement du « neutralisme », qui, à partir de la fin 1947, recherche «l'impossible troisième voie » comme une réaction européenne face aux deux blocs de la guerre froide.

\section{De l'affaire Tito à la fin de la guerre civile (juillet 1948- août 1949)}

17 Pendant cette période les thèmes abordés par la presse portent surtout sur le côté grec du conflit Tito-Staline. Quand la direction stalinienne du KKE dénonce Tito comme « traître » et se range aux côtés du Kominform dans sa campagne antititiste, Tito ferme ses frontières et porte un coup fatal à l'A.D. et aux communistes grecs. Au sein de la direction communiste de l'A.D., le désaccord entre Markos et Zachariadis, secrétaire général du KKE, aboutit au limogeage de Markos et à l'adoption par le KKE de la thèse en faveur d'un État macédonien autonome. Du coup, tandis que les communistes injurient Tito, des journaux anticommunistes traitent Markos avec une certaine sympathie, parce qu'il a osé s'élever contre les staliniens de son parti.

18 À partir de l'automne 1948, on se trouve devant la banalisation de la guerre civile qui occupe déjà l'actualité en permanence depuis presque trois ans. La presse, à l'exception du Monde et de l'Humanité, s'occupe de moins en moins des nouvelles venues de Grèce. La défaite de l'A.D., tout autant que la fin de la guerre, en août 1949, rencontre l'indifférence de la plupart des journaux.

\section{La Grèce éternelle}

19 Malgré sa profonde division, la presse est unanime pour manifester son affection envers les Grecs et leur pays. "Il y a une longue histoire entre la Grèce et nous », écrit Louis Aragon dans les Lettres françaises en février 1949. Pour les Français, la Grèce est familière. Il y a un rapport spécifique entre les deux pays, une tradition des rapports culturels et affectifs de longue date.

L'attitude de la presse est donc façonnée aussi par l'influence de l'image traditionnelle de la Grèce en France. En pleine guerre civile, ruinée et exsangue, la Grèce reste comme elle l'était toujours, la mère de la sagesse humaine, le berceau de la démocratie, le carrefour du monde où se rencontrent l'Occident et l'Orient, le rationnel et le mystique. Par ailleurs, la Grèce contemporaine est avant tout le petit pays héroïque dont la résistance antihitlérienne grandiose avait beaucoup contribué à la victoire alliée.

Je veux d'abord, avec toute mon admiration, tout mon amour, te saluer, Grèce claire et dure, diamant de l'Europe, Grèce de toujours, terre des héros, mère de la France. C'est toi qui nous as donné la civilisation, c'est toi qui nous as appris l'intelligence, la beauté, la mesure, le sourire et comment on lutte et on meurt pour la liberté ${ }^{12}$. 
21 Tout ce qui concerne le passé antique est respectable et digne d'admiration; au contraire, tout le négatif est lié à l'histoire grecque moderne et contemporaine. Les traits négatifs du caractère national grec sont jugés comme étant le revers de leurs vertus: les Grecs sont fiers, mais en même temps individualistes. Leur extrême politisation, leur " politicomanie ", se mue souvent en fanatisme. Leur héroïsme et leur amour de la liberté aboutissent à l'individualisme et à un "révolutionnarisme » excessif. Ce qui est cité dans un sens vraiment péjoratif, c'est la dimension balkanique de leur tradition historique, ainsi certains articles présentent-ils les Grecs de Macédoine comme enclins à la rébellion et à la sauvagerie.

La transition entre le mythe, les images idéalisées et la réalité grecque est parfois rude. Comme les philhellènes de la guerre d'indépendance de 1821 , les journalistes français qui arrivent en Grèce - dont la plupart revendiquent le titre de philhellènes - se trouvent face à une réalité qui les oblige à quitter leurs illusions.

Chez les Grecs l'image de la France est aussi idéalisée que celle que les Français, surtout les intellectuels. Confrontée à cette image de la France chez les Grecs, toute la presse française exprime la conviction que leur pays peut et doit élever sa voix pour que le drame grec se termine le plus tôt possible. Les envoyés spéciaux de tous les journaux racontent comment, à plusieurs reprises, s'est exprimé le profond attachement sentimental des Grecs pour la France :

Ma nationalité me sert près d'eux (des paysans dans un village en Thessalie) d'introduction et de mot de passe : nous évoquons Salonique et Verdun... ${ }^{13}$ Vous vous heurtez d'abord à la méfiance : puis vous dites : Gallos, Français, et alors les visages s'éclairent. Les Grecs pensent que la France est le pays de toutes libertés ${ }^{14}$.

24 La culture française et la connaissance de la langue française, très répandues et profondément enracinées dans la société grecque, sont considérées comme critère d'une bonne éducation et de reconnaissance sociale, même dans les couches petitesbourgeoises et paysannes :

Francophile la Grèce ? Elle est plus que cela! Il arrive qu'un paysan grec, devant doter sa fille, dise avec conviction au prétendant ambitieux : elle a tant de cheptel, tant d'ouvriers ; et puis, ne l'oubliez pas, elle a son piano et son français ${ }^{15}$.

25 L'image traditionnelle de la Grèce est utilisée pour créer de nouveaux mythes et symboles. Dans la presse anticommuniste, le petit pays héroïque devient le bastion de la lutte du monde libre contre la barbarie communiste. La presse communiste a trouvé une source nouvelle riche non seulement d'arguments propices à une utilisation politique, mais aussi d'images héroïques, de symboles et d'espoirs. Le PCF a vu dans les rudes andartès des montagnes les représentants d'un romantisme révolutionnaire cher à la culture communiste. Les nouveaux héros qui sont «les vrais descendants des guerriers de pierre archaïques et des héros légendaires d'Homère $»^{16}$. Journalistes et intellectuels communistes donnent de l'Armée démocratique l'image idéale d'une armée populaire qui se bat avec abnégation, du patriote communiste qui combat l'impérialisme américain.

26 L'attitude adoptée face à la guerre civile dans le cas de la gauche dans son ensemble au moins jusqu'à la fin 1947 - ne consiste pas simplement en une prise de position politique et idéologique, elle se manifeste également de manière concrète, aboutissant à la création d'un courant philhellénique et à celle d'un mouvement de soutien au peuple grec dans l'épreuve. 
La gauche non communiste retire son soutien politique aux communistes grecs, mais elle continue pourtant de dénoncer les répercussions contre la gauche en Grèce. Le premier noyau d'un comité gréco-français est créé dans l'été 1946. L'année suivante, un Comité de la région parisienne d'aide à la Grèce et un comité de défense des libertés du peuple grec sont constitués. En janvier 1948, le Comité français d'aide à la Grèce démocratique est officiellement fondé. Des personnalités de toute la gauche y participent, mais son contrôle politique effectif passe entre les mains des communistes et c'est la politique du PCF qui dicte ses orientations.

\section{Conclusion}

L'attitude de diverses tendances de la presse se forme en fonction des sensibilités politiques et affinités idéologiques et elle est absolument rattachée à l'évolution de la situation mondiale et de la vie politique française. D'après la logique de la guerre froide, les opinions exprimées se plient à des schémas simplistes où l'on oppose communisme et anticommunisme.

Les points les plus essentiels pourraient se résumer comme suit : l'attitude de diverses tendances de la presse se forme en fonction de l'idéologie et de ses choix politiques et elle est absolument rattachée à l'évolution de la situation mondiale et de la vie politique française. Les points de vue de chaque famille idéologique prennent naissance dans leur attitude globale vis-à-vis du nouvel ordre international, surtout après l'installation dans la guerre froide, après le mi-1947. Pour les deux camps opposés en France, communiste et anticommuniste, la guerre civile grecque constitue la preuve des plans criminels du bloc adversaire. En dernière analyse l'attitude de la presse est le miroir qui reflète la façon dont les Français vivent les premières années du grand schisme.

\section{BIBLIOGRAPHIE}

Sources

Tous les numéros des années 1946, 1947, 1948 et 1949 des quotidiens parisiens et des périodiques cités ci-dessous :

Quotidiens : L'Aube, L'Aurore, Ce Soir, Combat, La Croix, L'Époque, Le Figaro, Franc-tireur, France-Soir, L'Humanité, Libération, Le Monde, Paris-Presse, Le Parisien-Libéré, Le Populaire de Paris, La Vérité.

Périodiques et Revues : Action, Carrefour, Esprit, Gavroche, Les Lettres françaises.

De nombreux articles parus dans plusieurs périodiques et revues, ainsi qu'un grand nombre de brochures, documents officiels et textes littéraires contemporains de l'époque étudiée.

Pour une bibliographie sur les sujets convergents (la guerre civile grecque, l'Europe de l'aprèsguerre et la guerre froide, la vie politique et intellectuelle de la France de l'après-guerre, la 
presse française, l'étude de la presse et de l'opinion publique), voir : Athanassia Balta, L'opinion publique française face à la guerre civile grecque (1946-1949), op.cit., p. 341-354.

Pour un bilan de la production historiographique des deux dernières décennies sur la guerre

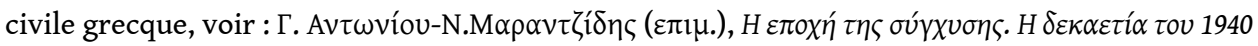

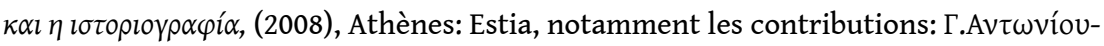

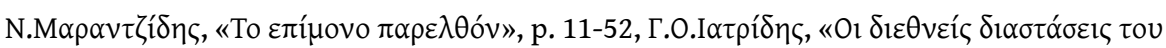

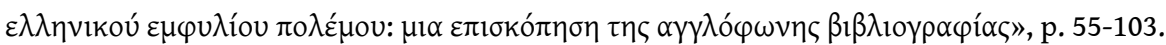

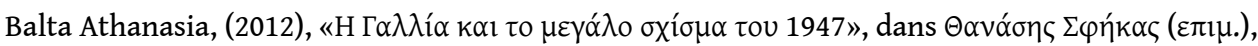

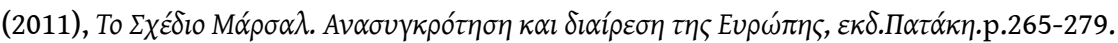

Berstein, Serge, Milza Pierre, (dir.), (2000), L'Année 1947, Paris : Presses de la Fondation des Sciences politiques.

\section{NOTES}

1. Dans cette communication je reprends les idées principales de ma thèse de doctorat: Athanasia Balta, L'opinion publique française face à la guerre civile grecque (1946-1949), Thèse de doctorat de $3^{\mathrm{e}}$ cycle en histoire $\mathrm{du} \mathrm{xx}^{\mathrm{e}}$ siècle,(non publiée), dirigée par Pierre Milza, Institut

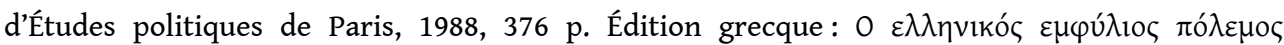

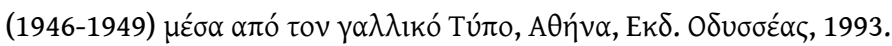

2. Le Monde, bulletin de l'étranger, 11 avril 1946.

3. Le conflit Tito-Staline, qui a bouleversé le monde communiste, elle avait aussi des conséquences lourdes pour l'A. D. et pour l'issue de la guerre civile.

4. L'Humanité, « Voici nous nous battons », 30-31 mars 1947.

5. Abréviations

A.D. Armée démocratique,

C.F.A.G.D. Comite Français d'aide à la Grèce démocratique,

EAM Ethniko Apelevtherotiko Metopo, Front National de Libération,

KKE Kommounistiko Komma Ellados, Parti Communiste de Grèce,

PCF Parti Communiste Français,

UNSCOB United Nations Spécial Commitee on the Balkans.

6. Le Populaire de Paris, 27 octobre 1947.

7. Le KKE avait déclaré son projet de créer un gouvernement provisoire en "Grèce Libre » au $\mathrm{XI}^{\mathrm{e}}$ Congrès du PCF, réuni à Strasbourg, en juin 1947, par son représentant Porphyrogenis. Cette déclaration, passée "discrètement » dans l'Humanité en $5^{\mathrm{e}}$ page, témoigne, entre autres indices, du fait que le PCF, malgré sa position officielle, est réservé face à la politique du KKE. La publicité faite au GDP six mois plus tard par la presse communiste s'explique par la politique du PCF après son départ du gouvernement et la formation du Kominform.

8. Le Monde, bulletin de l'étranger, 26 décembre 1947.

9. L'Humanité, 28-29 décembre, reportage intitulé «Je t'ai retrouvée, Grèce des héros, mère de la France ».

10. L'Humanité, 21 mars 1946, article de Marcel Cachin, « En Grèce, en Espagne ».

11. L'Aurore, 11 juillet 1947.

12. Simone Téry, Ils se battent aux Thermopyles, 1948, p. 229.

13. Action, 17 janvier 1947, reportage de J. Grégoire.

14. Gavroche, 22 août 1946.

15. Le Figaro, 3 juin 1947, reportage de Dominique Auclères.

16. Action, 24 février 1949. 


\section{RÉSUMÉS}

Quel intérêt la presse française a-t-elle porté à la guerre civile grecque à un moment où le pays devait lui-même se rétablir? Comment a-t-elle interprété cet événement et l'a-t-elle présenté à l'opinion publique? On peut distinguer trois étapes, début 1946-mars 1947, mars 1947-juin 1948 et juillet 1948-août 1949. On voit que l'attitude des diverses tendances se forme en fonction de l'idéologie et des choix politiques et qu'elle est rattachée à l'évolution de la situation mondiale et de la vie politique française. Chaque famille idéologique juge selon son attitude vis-à-vis du nouvel ordre international, surtout après l'installation dans la guerre froide. Pour les deux camps opposés, communiste et anticommuniste, la guerre civile grecque constitue la preuve des plans criminels du bloc adversaire. La presse reflète donc la façon dont les Français vivent les débuts de la guerre froide.

What kind of interest did the French press bear to the Greek Civil War in a moment when the whole country had to be restored? How did it interpret the event and present it to public opinion? One can distinguish three moments, from the beginning of 1946 to March 1947, from Mars 47 to June 48 , and from July 48 to August 49 . One can observe that the attitude of the various trends is formed according to the ideology and political choices and that it is related to the evolution of world public opinion as to the french political life. Each ideological family judges according to its attitude towards the new international order, mainly after the beginning of the Cold War. For both opposing camps, communist and anticommunist, the Greek Civil War is the evidence of the criminal plans of the opposing bloc. The press therefore reflects the way the French people live the beginning of the Cold War.

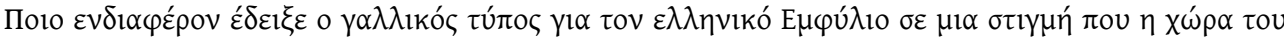

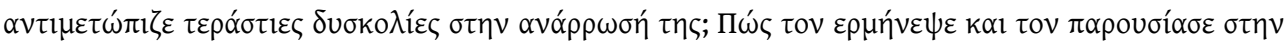

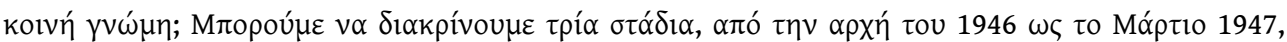

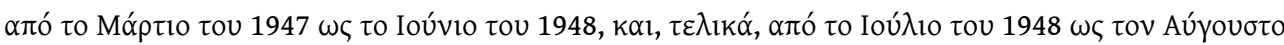

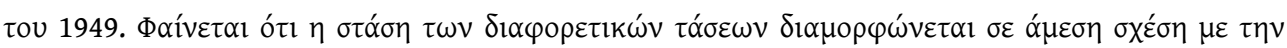

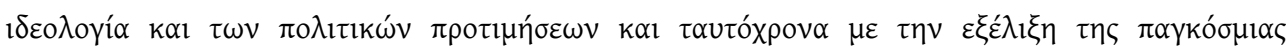

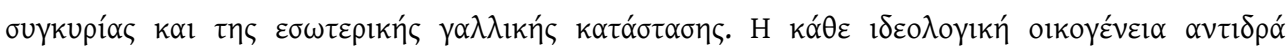

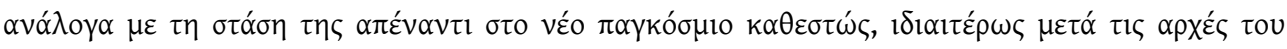

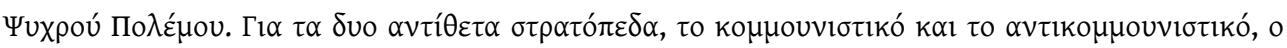

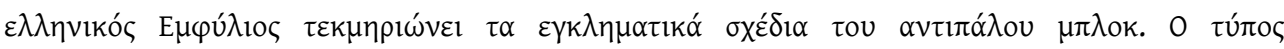

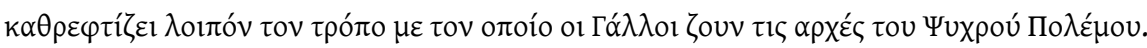




\section{INDEX}

motsclestr Fransız basını, Bilgi, Public görüş, Propaganda, Marshall Planı, Truman doktrini, Cominform, Vafiadis Markos (1906-1992), Zahariadis Nikos (1903-1973), Fransa, Yunanistan, Yunan iç savaş, Soğuk harp, Tarih, Basın Tarihi

Mots-clés : presse française, information, Andartes, opinion publique, Georges II (1890-1947), propagande, plan Marshall, doctrine Truman, Kominform, Vafiadès Markos (1906-1992), Vafiadès Markos (1906-1992), Zachariadis Nikos (1903-1973), Zachariadis Nikos (1903-1973)

motsclesmk ФРАНЦУСКИОТ ПЕЧАТ, ИНФОРМАЦИИ, МИСЛЕЊЕ ЈАВНОСТА, ПРОПАГАНДА, МАРШАЛОВ ПЛАН, ТРУМАН ДОКТРИНА, ИНФОРМБИРОТО, ВАФИАДИС МАРКОС (1906-1992), ЖАХАРИАДИС НИКОС (1903-1973), ФРАНЦИЈА, ГРЦИЈА, ГРЧКАТА ГРАЃАНСКА, СТУДЕНАТА ВОЈНА, ИСТОРИЈА, ИСТОРИЈА НА ПЕЧАТОТ

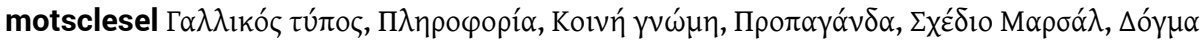

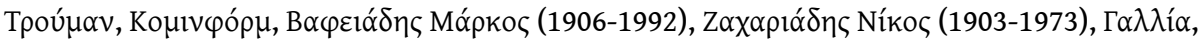

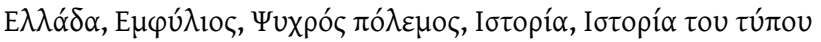

Thèmes : Histoire, Histoire de la presse

Index chronologique : guerre froide, guerre civile grecque (1946-1949)

Keywords : information, public opinion, propaganda, Truman doctrine, Cominform, Vafiadès Markos (1906-1992), Zahariadis Nikos (1903-1973), France, Greek Civil war, Cold war, History 